\title{
Fitness-based Neighbor Selection for Multimodal Function Optimization
}

\author{
Shin Ando \\ Yokohama National University \\ Yokohama, Kanagawa, Japan \\ ando@slab.dnj.ynu.ac.jp
}

\author{
Shigenobu Kobayashi \\ Tokyo Institute of Technology \\ Yokohama, Kanagawa, Japan \\ kobayashi@fe.dis.titech.ac.jp
}

\begin{abstract}
We propose a selection scheme called Fitness-based Neighbor Selection (FNS) for multimodal optimization. The FNS is aimed for ill-scaled and locally multimodal domain, both found in real-world numerical optimization problem.

In FNS, selection is applied to parent-child pair that most likely belong to the same attractor. We determine such pair with statistical comparison of the fitness values sampled from region between the pairs, instead of conventional Euclidean distance. In addition, the ranks of a parent among sampled values are used to determine if the parent is replaceable. These measurements makes the algorithm scaleinvariant thus robust in ill-scaled domain.

Categories and subject discriptors: Computing Methodologies[Artificial Intelligence]:Problem Solving, Control Methods, Heuristic Methodsand Search
\end{abstract}

General Terms:Algorithm

Keywords:Genetic Algorithm, Multimodal Optimization, Niching

\section{INTRODUCTION}

The general purpose of Multimodal optimization (MO) is to obtain multiple optima/suboptima comprehensively or to certain extent. MO can provide practical soultion in many instances of real-world problems where preparing multiple solutionswith varying feature is a better strategy. Many researches have successfully applied modified selection procedure, also called Niching methods, to MO tasks [1, 4, 5, $2]$.

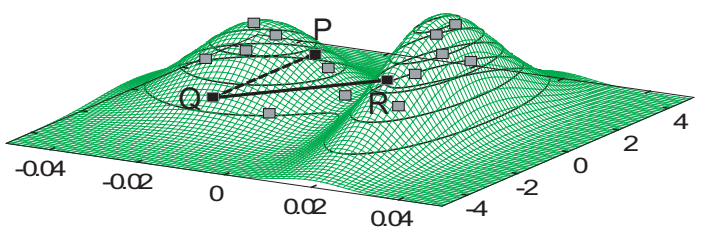

Figure 1: Pop. Snapshot on Ill-scaled Attractors

Copyright is held by the author/owner.

GECCO'05, June 25-29, 2005, Washington, DC, USA.

ACM 1-59593-010-8/05/0006.
However, these conventional methods do not assume illscaled domains, and commonly report difficulty in locally multimodal domains. Ill-scaling and local multimodality are commonly found in real-world problems, and present difficulty for most optimization algorithm.

\section{PROBLEM DOMAIN}

We will refer to the convex around a suboptima as its attractor. In Fig.1, $P, Q$ are on one attractor and $R$ is on another.

In the ill-scaled domain, some variables are significanlty sensitive to fitness function than others (See scaling in Fig.1), or have strong correlations. This is problematic for methods using Euclidean distance. In Fig.1, it is difficult to decide parameters such as sharing distance[2] or minimum distance to between 'species' $[4,1]$. Generally, individuals on two attractors are difficult to distinguish with Euclidean distance, which can negate the Niching methods.

Locally multimodal domains have concentrated 'clusters' of suboptima. Reportedly, finding suboptima within a 'cluster' is commonly difficult for Niching methods[4].

\section{IMPLEMENTATION}

We propose a selection scheme called Fitness-based Neighbor Selection (FNS). FNS apply selection to parent-child pair who are likely neighbors. However, instead of Euclidean distance, the neighboring pair is determined by statistical comparison of the sampled fitness values. Further, the ranks of a parent's fitness among the samples is used to measure the parent's replaceability. Above measurements are scale invariant, giving FNS robustness in the ill-scaled domain.

\subsection{Genetic Operators / Replacement Scheme}

FNS uses ENDX[3] crossover, an extension of UNDX[6]. It defines normal probability distribution from the parents to create offspring. It is successful in many benchmarks.

Following are the procedures for one cycle of replacement scheme, which is a modification of Minimal Generation Gap (MGG) [7]. Step 3 is detailed in Section 3.2.

1. Randomly select $m$ parents from population $P$.

2. Generate $\lambda$ offspring by iterated crossover.

3. Apply selection to best child and neighboring parent(s).

\subsection{Fitness-based Neighbors}

In Fig.1, $P, Q$ are neighbors intuitively, while $R$ are not, as latter is on a different attractor. More mathematically, we consider the mean fitness $M$ over path $P Q$ and $Q R$.

$$
M_{P Q}=\int_{P Q} f(\mathbf{x}) d \mathbf{x} / \int_{P Q} d \mathbf{x} \quad(f(x): \text { FitnessFunction })
$$

If $M_{P Q}$ is significantly better than $M_{Q R}$, the pair is more 

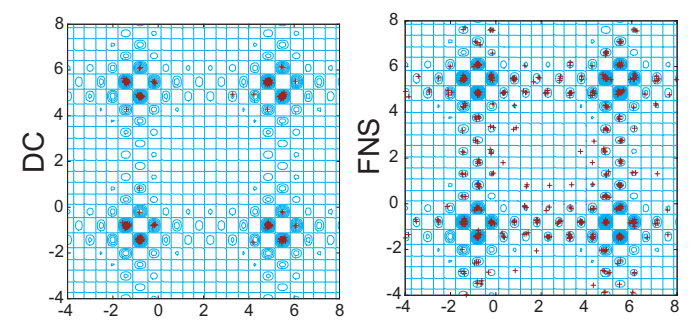

Figure 2: FNS and DC Snapshot on $F_{1}$

likely to be on the same attractor than the other. Since the integral of fitness function is usually uncalculable, it is practical to compare sampled fitness values. We use fitness values $\mathbf{f}_{P Q}$, sampled from normal distribution around the path $P Q . \mathbf{f}_{P Q}$ can be obtained by crossover of $P, Q$, and recording fitness of the offspring. We compare mean fitness $\overline{\mathbf{f}}_{P Q}$ and $\overline{\mathbf{f}}_{Q R}$ using Wilcox's Rank-sum Test.

FNS performs the above after each crossover on best child $c$ and two parents $p_{0}, p_{1}$. First, we crossover $\left(p_{0}, c\right)$ to sample fitness values $\mathbf{K}_{0}$, then $\left(p_{1}, c\right)$ for $\mathbf{K}_{1} . K_{0} \gg K_{1}$ denote $\bar{K}_{0}$ is better than $\bar{K}_{1}$ with significance level $\alpha$ in Rank-Sum Test. Tournament selection is applied to $c$ and $p_{i}$ if $K_{i} \gg K_{j}$.

Further, we measure replaceability of parents $p_{i}$ by its rank. $R_{i}$ denote the rank of $p_{i}$ 's fitness among $\mathbf{K}_{i} . p_{i}$ is not subject to replacement if $R_{i}$ is above threshold $R_{t h}$. This prohibits the replacement of parents on a third attractor.

The summary of crossover and FNS selection scheme:

1. Crossover $p_{0}, p_{1}, \ldots, p_{m}$ and select best offspring $c$.

2. Crossover $p_{0}, c, \ldots, p_{m}$ to samples fitness values $\mathbf{K}_{0}$.

3. Crossover $c, p_{1}, \ldots, p_{m}$ to sample fitness values $\mathbf{K}_{1}$.

4. If $\mathbf{K}_{\mathbf{0}} \ll \mathbf{K}_{\mathbf{1}}$, apply tournament selection to $\left(p_{1}, c\right)$.

5. If $\mathbf{K}_{\mathbf{0}} \gg \mathbf{K}_{\mathbf{1}}$, apply tournament selection to $\left(p_{0}, c\right)$.

6. Otherwise, consider ranks $R_{0}, R_{1}$.

In step 6, elitist selection is applied to replaceable parent(s) and $c . p_{i}$ is replaceable when $R_{i}>R_{t h}$. The replacement does not occur if neither parents are replaceable.

\section{EXPERIMENTS}

We compare Deterministic Crowding (DC) [5] and FNS with two benchmarks with properties decribed in Section2.

\subsection{Locally Multimodal Domain}

Shubert Function is often used as locally-multimodal benchmark[4]. Within the range $\left(-4 \leq x_{i} \leq 8\right)$, it has over 100 suboptima distributed symmetrically. There are $2^{n}$ pairs of optima, each pair within a cluster of $4^{n}$ suboptima. Fig.2 shows its contour by light lines.

FNS and DC are applied to 2-D $F_{1}$, using population size of 500 for 30,000 steps. The offspring size $\lambda=4$. FNS parameters are SampleSize $=100, R_{t h}=0.5$, and $\alpha=0.1$.

Fig. 2 shows FNS and DC populations after 30,000 selection from a typical run with dark ' + '. DC repeatedly replaced parents on suboptimal attractors when nearest offspring is created on nearby optimal attractors in the 'cluster'. As a result, final population occupy optimal attractors and few strong suboptimal attractors. Meanwhile, FNS maintain population on suboptimal attractors, with consitent analysis of the neighbors by sampled values and ranks.

\subsection{Ill-scaled Domain}

Next, we evaluate FNS in a non-linear, ill-scaled function $F_{2}$, derived from Rosenbrock's function (1). $F_{2}(2)$ has a parabolic dominant attractor and a weaker attractor placed parallel to each other. The optimal value of $F_{2}(\vec{x})=0$ is
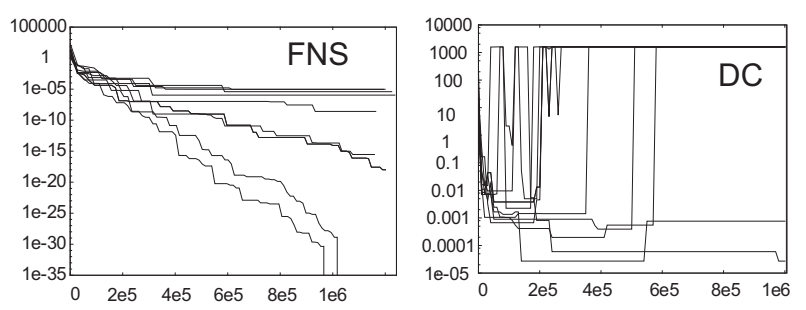

Figure 3: Best fitness value on suboptimal attractor

found along the dominant 'optimal' attractor and at a point $(1,1)$ in weaker 'suboptimal' attractor.

$$
\begin{gathered}
F_{R}(\vec{x})=\sum_{i=2}^{n}\left\{100\left(x_{1}-x_{i}^{2}\right)^{2}+\left(x_{i}-1\right)^{2}\right\} \\
F_{2}(\vec{x})=F_{R}(\vec{x}) \times \sum_{i=2}^{n}\left\{100\left(x_{1}-\left(x_{i}-4\right)^{2}\right)^{2}\right\}
\end{gathered}
$$

We assess FNS and DC's ability to search a weak attractor by tracking $F_{R}(y)$ for generation's best individual $y . F_{R}(y)$ is roughly the best fitness found in suboptimal attractor.

We run FNS and DC 10 times, each for 1,000,000 evaluations, using popsize 40 and offspring size $\lambda=100$. FNS parameters are the same from previous experiment.

Fig. 3 shows the convergence of $F_{R}(y)$ for 10 runs of DC and FNS. DC runs show increase in fitness value, which indicates that best individual in suboptimal attractor was replaced by one in the optimal attractor. In 8 out of 10 runs, suboptimal attractor was completely abandoned. Meanwhile, FNS maintained steady improvement at varied convergence speed depending on the number of individuals on the suboptimal attractor. It reached the exact optima $(1,1)$ within the observed timeframe in two runs .

\section{CONCLUSION}

FNS have shown qualitative advantage in ill-scaled and locally multimodal domain. This makes FNS practical for real-world problems, and compensate for its apparent tradeoff of increased evaluation for sampling.

Like other Niching methods, FNS will show loss of convergence speed in highly multimodal domain. Effective combination of FNS with sophisticated reproductive selection scheme is in the future works.

\section{REFERENCES}

[1] K. Deb and D. E. Goldberg. An investigation of niche and species formation in genetic function optimization. In Proceedings of the Third ICGA, pages 42-50, 1989.

[2] D. E. Goldberg and J. Richardson. Genetic algorithms with sharing for multimodal function optimization. In Proceedings of the Second ICGA, pages 41-49, 1987.

[3] S. Kimura, I. Ono, H. Kita, and S. Kobayashi. in japanese. Transactions of the Society of Instrument and Control Engineers, 36:1162-1171, 2000.

[4] J. Li, M. Balazs, and P. Clarkson. A genetic algorithm using species conservation for multimodal function optimization. Evolutionary Computation, 10(3), 2002.

[5] S. W. Mahfoud. Crowding and preselection revisited. In Proceedings of PPSN II, pages 27-36, 1992.

[6] I. Ono and S. Kobayashi. A real-coded genetic algorithm for function optimization using unimodal normal distribution crossover. In Proceedings of 7th ICGA, pages 246-253, 1997.

[7] H. Satoh, M. Yamamura, and S. Kobayashi. Minimal generation gap model for gas considering exploration and exploitation. In Proceedings of IIZUKA, 1996. 